\title{
Role of membrane glycoproteins in mediating trophic responses
}

\author{
R TAUBER, W REUTTER, AND W GEROK \\ Medizinische Klinik der Universität Freiburg, Freiburg i Br, Federal Republic of Germany, and \\ Institut für Molekularbiologie und Biochemie der Freien Universität, Berlin, FRG
}

SUMMARY During growth and differentiation the plasma membrane has a key role not only in the reception and transmission of extracellular signals such as hormones and growth factors, but also in communicating cellular response to the cellular microenvironment. Cellular response to trophic stimuli includes alterations of cell shape and cell surface antigenicity, ${ }^{1}$ of cell-cell recognition and cellular adhesion, ${ }^{2}$ of cell matrix binding ${ }^{3}$ and the adaptation of cell surface receptors. ${ }^{4}$ The plasma membrane is therefore regarded as a 'central agency' for the integration of a single cell into the complex system of a tissue or of an organism. The numerous functions of the plasma membrane are mainly mediated by membrane integrated glycoproteins or glycolipids both sharing the common feature of covalently bound oligosaccharide side chains. Specific alterations of oligosaccharide structure and metabolism associated with growth, differentiation and various pathologic conditions suggest a specific role for the oligosaccharide moieties in the regulation of cell surface functions (Table 1). This review intends to focus on the role of plasma membrane glycoproteins describing briefly principles of glycoprotein structure and function, and characteristics of their biosynthesis and degradation.

\section{Structure of plasma membrane glycoproteins}

The polypeptide backbone of plasma membrane glycoproteins is constituted by at least two domains, a sequence rich in hydrophobic amino acids which anchors them to the lipid bilayer, and a hydrophilic domain at the extracellular membrane surface. Several glycoproteins such as the insulin receptor, the EGF receptor or the LDL receptor span the membrane and

Table 1 Alterations of oligosaccharide structures of plasma membrane glycoproteins

\begin{tabular}{ll}
\hline & Selected references \\
\hline Differentiation & 5 \\
Growth & 67 \\
Mutation & 8 \\
Hypervitaminosis (retinol) & 910 \\
Malignancy & 1112 \\
Cystic fibrosis & 13 \\
Psoriasis & 14 \\
\hline
\end{tabular}

Address for correspondence: Prof R Tauber, Institut für Klinische Chemie und Biochemie, Universitatsklinikum Charlottenburg, Spandauer Damm 130, D-1000 Berlin 19, FRG

This study is dedicated to Prof P Schölmerich on the occasion of his 70th birthday have a third cytoplasmic domain. ${ }^{15}$ The membrane anchor sequence of transmembrane glycoproteins is flanked by basic sequences which may interact with the head groups of negatively charged phospholipids. ${ }^{15}$

Oligosaccharide side chains of glycoproteins are exclusively bound to the extracellular polypeptide domain extending into the microenvironment of the cell. Structural analysis by use of 360 and $500 \mathrm{MHz}$ 'H-NMR or sequential exoglycosidase digestion has shown that oligosaccharides of glycoproteins fall into two classes according to the type of their carbohydrate polypeptide linkage (Fig. 1, ${ }^{16}$ ) (a) O-glycosidic linkage from $\mathrm{N}$-acetyl-D-galactosamine to hydroxyamino acids (serine, threonine), (b) $\mathrm{N}$-glycosidic linkage from $\mathrm{N}$-acetyl-D-glucosamine to the amide nitrogen of asparagine.

Asparagine linked oligosaccharides have a common core structure consisting of a branched pentasaccharide Man $\alpha 1-3$ (Man $\alpha 1-6)$ Man $\beta 1-$ 4GlcNAc $\beta$ 1-4 GlcNAc-Asn. To the peripheral mannose residues different types of side chains are linked giving rise to three structural subgroups. High mannose or oligomannosyl oligosaccharides are substituted with additional mannose residues, whereas 


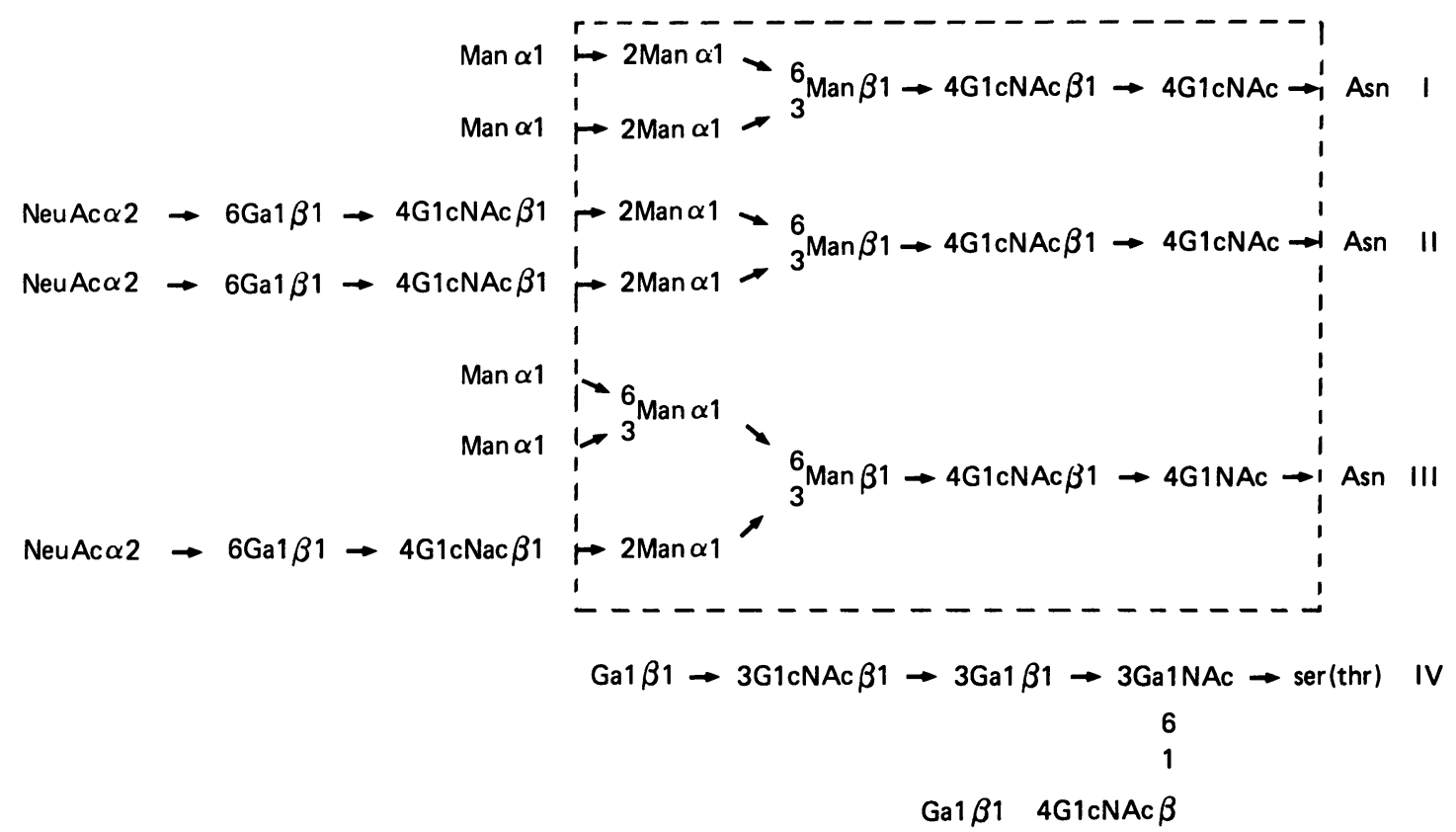

Fig. 1 Representative structures of $N$-linked and $O$-linked oligosaccharide chains. I: High mannose type, II: complex type, III: hybrid type of N-linked oligosaccharides; IV:O-linked structure. Fuc, L-fucose; Gal, D-galactose; GalNAc, N-acetyl-D-galactosamine; GlcNAc, N-acetyl-D-glucosamine; Man, D-mannose; NeuAc, N-acetylneuraminic acid.

complex type oligosaccharides contain two, three, four or five outer branches consisting of one lactosamine sequence $\mathrm{Gal} \beta 1-4 \mathrm{GlcNAc}$ or repeating lactosamine units. To the galactose or $\mathrm{N}$-acetyl-D-glucosamine residues of the lactosamine sequence $\mathrm{L}$-fucose or $\mathrm{N}$-acetylneuraminic acid may be linked as terminal substituents. Additionally, L-fucose may be linked to the C-6 position of the innermost N-acetyl-Dglucosamine residue. Polysialosyl sequences with up to 12 sialic acid residues have been found in rat brain. ${ }^{17}$ Thirdly, hybrid oligosaccharides share the feature of both high mannose and complex type oligosaccharides containing both oligomannosyl and lactosamine side chains.

By varying the sugar composition of the outer chains, the degree of branching and the type of the glycosidic linkages a tremendously high number of different oligosaccharides structures may be generated. Nevertheless, except for a certain microheterogeneity, the individual glycosylation sites of a glycoprotein have a high selectivity for a particular oligosaccharide structure indicating that oligosaccharide biosynthesis must be specifically regulated.

Unlike N-linked oligosaccharides O-glycosyl units have no common partial structure varying from disaccharides to complex branched oligosaccharides often attached to a Gal $\beta 1-3$ GalNAc disaccharide core. In A, B, and $\mathrm{H}(\mathrm{O})$ blood group determinants galactose, L-fucose and $\mathrm{N}$-acetyl-D-galactosamine residues are bound to this disaccharide in different positions.

The structural diversity of oligosaccharides is extended by their spatial conformation (for review see. ${ }^{18}$ ) Biantennary oligosaccharides - for example, may form three dimensional structures shaped like a $\mathrm{T}$ or $\mathrm{Y}$ thus exposing also sugar sequences in an inner position. Formation of three dimensional structures involves mutual interactions with the protein moiety and is therefore subject also to alterations of the amino acid sequence. Moreover, removal of terminal neuraminic acid residues forming non-covalent bonds with basic amino acids of the polypeptide, may modify the conformation of the oligosaccharide. Because glycosidic linkages are partly able to rotate, oligosaccharides of glycoproteins must be regarded as flexible structures.

\section{Functions of plasma membrane glycoproteins}

Because of their structural diversity and modifiability $\mathrm{O}$ - and N-linked oligosaccharides of plasma membrane glycoproteins serve as carriers of biological information either modulating the properties of functional glycoproteins or serving as specific signals 
Table 2 Functions of the oligosaccharide moiety of glycoproteins

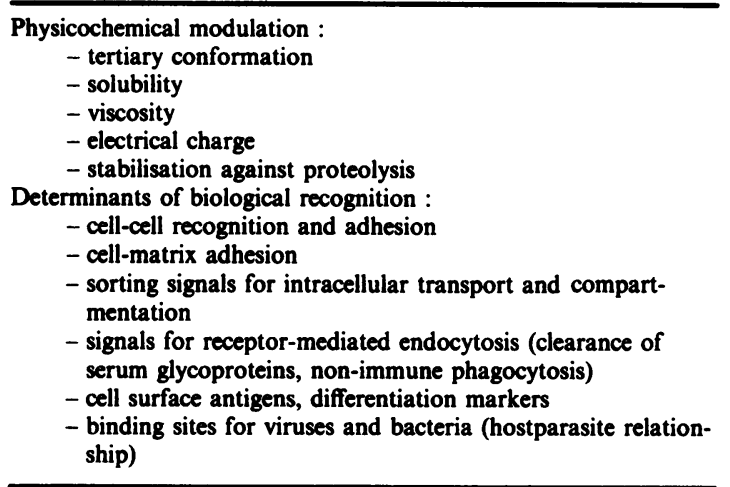

in numerous recognition systems (Table 2, for review). ${ }^{19,20}$

\section{PLASMA MEMBRANE GLYCOPROTEINS IN} INTERCELLULAR RECOGNITION AND ADHESION The ability of cells to recognise and to bind to each other specifically is a prerequisite for the development of multicellular organisms. Intercellular communication moreover plays pivotal roles in fertilisation, cellular differentiation, organogenesis, and in both adaptive and malignant growth.

Evidence that carbohydrate moieties of plasma membrane glycoproteins are crucial for intercellular communication has been obtained in several cellular systems (Table 3). Sugar or oligosaccharide determinants serving as recognition markers or binding sites have been partly characterised using four major experimental approaches: (1) studies in cell mutants with genetic defects in glycoprotein biosynthesis, ${ }^{23}(2)$ specific inhibition of glycosylation by drugs and antimetabolites, ${ }^{31}$ (3) modification of oligosaccharide biosynthesis by glucosidase inhibitors ${ }^{31}$ and (4) removal of single sugar residues by specific glycosidases. Especially terminal sugar sequences of complex $\mathrm{N}$ linked oligosaccharides have been described as im- portant signals - for example, in interce!lular adhesion. ${ }^{23}$ Treatment of BHK cells with neuraminidase exposing terminal $\beta$-galactosyl residues has been shown to increase cellular aggregation, whereas additional removal of the galactose residue decreases the aggregation potential of the cells. ${ }^{23}$ Similarly, a switch in glycoprotein biosynthesis from complex type oligosaccharides to high mannose structures results in lower cell-cell aggregation. ${ }^{23}$

As first suggested by Roseman, ${ }^{32}$ surface located oligosaccharides of plasma membrane glycoproteins or glycolipids are thought to mediate cellular recognition and adhesion by binding to complementary binding sites exposed on the surface of adjacent cells. This conception originally proposed for cell surface glycosyltransferases was restored to prominence by the discovery by Ashwell of cell surface receptor proteins with binding specificity for mono- and

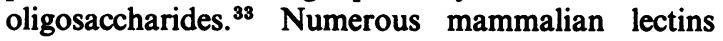
with different carbohydrate specificity have been characterised on the surface of various cell types (for review see. ${ }^{34-36}$ ) Figure 2 schematically shows how binding of a complex $\mathrm{N}$-linked oligosaccharide to a galactose specific lectin is controlled by the terminal sugar sequence. Binding to the lectin is initiated by the removal of terminal neuraminic acid which masks the penultimate galactose residue, whereas segregation of the oligosaccharide from the receptor may result from the subsequent removal of the galactose determinant. Desialylation of serum glycoproteins followed by binding and endocytosis by a galactose specific hepatic lectin has been characterised in detail as a major mechanism of the regulation of serum glycoprotein homeostasis. ${ }^{34}$ The identification of developmentally regulated lectins which are prominent at a specific stage of development of a tissue, ${ }^{36}$ and of tumour associated lectins ${ }^{37}$ indicates that oligosaccharide-lectin interactions participate in embryonic development and in malignant growth. Structural modifications of cell surface oligosaccharides observed in developing ${ }^{5}$ and neoplastic cells ${ }^{1112}$ could represent the de novo synthesis of lectin binding sites

Table 3 Oligosaccharides of plasma membrane glycoproteins in intercellular recognition

\begin{tabular}{llll}
\hline Function (cell type) & Sugar determinant & Selected reference \\
\hline Cell-cell binding & & 21 \\
Dictyostelium disc & N-Acetyl-D-galactosamine & 2223 \\
Fibroblasts & N-Acetylneuraminic acid & 24 \\
& D-Galactose & L-Fucose & 2526 \\
Intestinal epithelium & L-Fucose & 2728 \\
Lymphocyte homing & N-Acetylneuraminic acid & 2930 \\
Sequestration of & D-Galactose & D-Galactose, L-Fucose \\
erythrocytes & N-Acetyl-D-galactosamine & \\
\hline
\end{tabular}




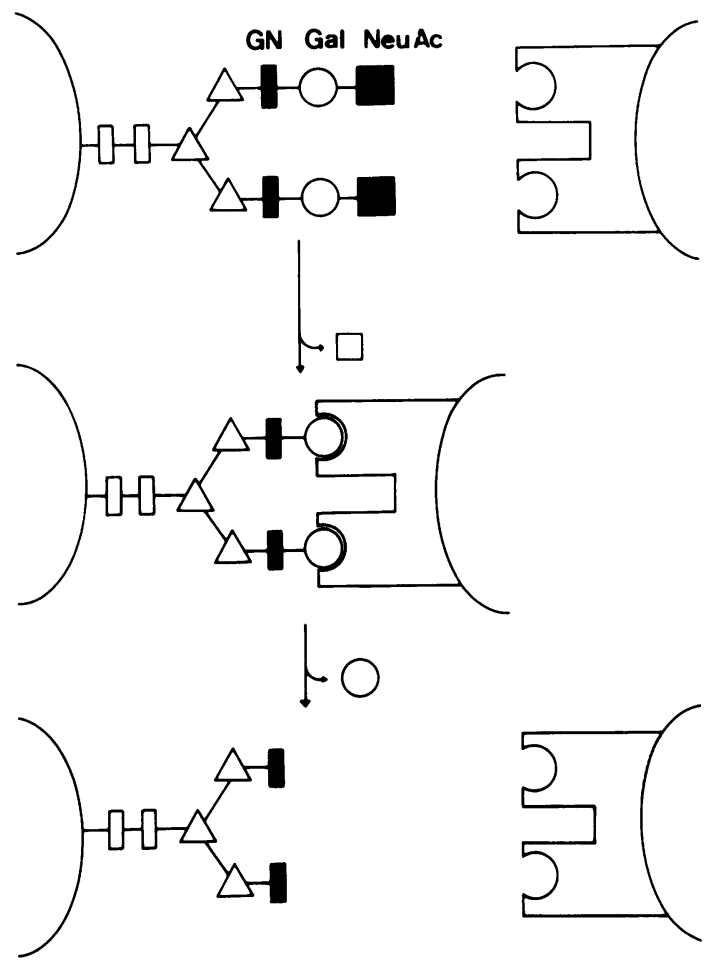

Fig. 2 Schematic model of oligosaccharide lectin interaction. Details see text.

or the masking and unmasking of preformed ones. In several forms of disease alterations of cell surface oligosaccharides correlate with defects in adhesion $;^{13}$ the molecular mechanisms, however, are not yet characterised. Apart from glycoprotein lectin binding the complex process of intercellular recognition and adhesion involves specific 'cell adhesion molecules', ${ }^{38}$ elements of the cytoskeleton ${ }^{39}$ and multifunctional glycoproteins loosely attached to the extracellular surface of the plasma membrane such as fibronectin. ${ }^{40}$

\section{CELL SURFACE ANTIGENS}

As shown by the use of monoclonal antibodies carbohydrate structures of glycoproteins and glycolipids are prominent antigens and constitute almost all cell surface associated antigens of the onco developmental type that have been characterised so far. ${ }^{4142}$ Antigens expressed at different stages of differentiation or during neoplasia often differ in single terminal sugar residues linked to a common saccharide backbone, as shown for a family of blood group related antigens based on the carbohydrate backbone sequence Gal $\beta 1-4(3)$ GlcNAc. ${ }^{1}$ Hence, changes in antigenicity during embryogenesis, differ- entiation or neoplasia may be a corollary of changes in the glycosylation of plasma membrane glycoproteins or glycolipids.

\section{OLIGOSACCHARIDES OF CELL SURFACE RECEPTORS}

$\mathrm{N}$ - and O-linked oligosaccharides are associated with most, if not all cell surface receptors. Although still a matter of inconsistency, there is increasing evidence supporting the view that intact glycosylation is required for biosynthesis and assembly of receptor oligomeric structures, for the stability of receptors after insertion into the plasma membrane and for the control of binding affinity. Inference has been mostly drawn from studies using tunicamycin, an antibiotic which inhibits the biosynthesis of $\mathrm{N}$-linked oligosaccharides, ${ }^{31}$ and from cell mutants with defects of glycosylation. ${ }^{4344}$ Glycosylation has been shown to be an essential step in the biosynthesis of the insulin receptor $^{45}$ and the acetylcholine receptor. ${ }^{4647}$ Like other glycoproteins receptors are synthesised in membrane bound polysomes of the rough endoplasmic reticulum, glycosylated cotranslationally and routed via the Golgi apparatus to the plasma membrane (for review see ${ }^{4849}$ ). The $\alpha$ - and $\beta$-subunits of the heterotetrameric insulin receptor derive from a single polypeptide precursor which is proteolytically processed to the precursors of the mature subunits post-translationally during intracellular transport. After treatment of cells with tunicamycin the nonglycosylated proreceptor is incapable of undergoing processing and is not transported into the plasma membrane ${ }^{45}$ Similarly, inhibition of glycosylation seems to inhibit transport and assembly of acetylcholine receptor subunits followed by a rapid decay of cell surface ligand binding activity. ${ }^{4647}$ Conversely $\mathrm{N}$-linked oligosaccharides do not play a major role in the biosynthetic routing of the asialoglycoprotein receptor pointing to individual differences of receptors in their glycosylation requirements. ${ }^{50}$

Second to biosynthesis the concentration of a receptor at the cell surface can also be regulated by interiorisation of the receptor followed by either degradation ${ }^{51}$ or delivery to an intracellular storage compartment. ${ }^{52}$ Although the biochemical mechanisms that control receptor down regulation are not yet known, evidence is accumulating that the oligosaccharide moiety has a key role in the regulation of receptor glycoprotein degradation. Lack of $\mathrm{N}$-linked oligosaccharides or O-linked oligosaccharides causes enhanced degradation of the acetylcholine receptor ${ }^{53}$ or the LDL receptor, ${ }^{43}$ respectively. Furthermore, studies in cell mutants suggest that not only deficiency but also structural alterations of $\mathrm{N}$ - and $\mathrm{O}$-linked oligosaccharides may increase receptor breakdown. ${ }^{44}$ As oligosaccharides of plasma membrane glyco- 
proteins are stepwise degraded either in situ at the cell surface or during membrane recycling ${ }^{54-56}$ alterations of oligosaccharide structures are likely to occur during the life cycle of a receptor glycoprotein. Intact glycosylation may be essential for stabilisation of the receptor molecule against proteolysis (for review $\mathrm{see}^{20}$ ) or against denaturation in the acidic environment of endosomes, or may be part of an escape mechanism that protects receptors from segregation into the lysosomal compartment. Influencing protein conformation, accessibility of binding sites or the proper exposure of the receptor on the cell surface, structural carbohydrates may also modulate receptor binding affinity.

According to these examples, oligosaccharides of plasma membrane glycoproteins are involved in cell surface functions in at least two different ways: (1) either as effectors - for example, when interacting with sugar specific receptors in cell-cell recognition, or (2) as covalently-bound modulators of activities effected by the polypeptide moiety - for example, in receptors. Both of these mechanisms are subject to structural alterations of the oligosaccharide side chains. In order to avoid uncontrolled structural changes hazardous for cell surface functions, but also to generate specific structures, which may modulate a particular function, oligosaccharides of plasma membrane glycoproteins must be under precise control.

\section{Biosynthesis and degradation of plasma membrane glycoproteins}

In contrast with the other polymers of the cell DNA, RNA and proteins, oligosaccharides are not synthesised on a template. Biosynthesis of $\mathrm{N}$-linked oligosaccharides ${ }^{49}$ starts in the endoplasmic reticulum with the assembly of a common precursor oligosaccharide $\mathrm{Glc}_{3} \mathrm{Man}_{9}\left(\mathrm{GlcNAc}_{2}\right)$ linked by pyrophosphate to the lipid carrier dolichol. Catalised by specific glycosyltransferases monosaccharide residues are stepwise transferred to the lipid carrier from either sugar nucleotides or dolichol-linked sugars. The precursor oligosaccharide which contains the common pentasaccharide core of $\mathrm{N}$-linked oligosaccharides is highly conserved in evolution and found in nearly all eukaryotes. After en bloc transfer to asparaginyl residues that are part of a Asn-X-Ser/ Thr sequence, the precursor oligosaccharide is extensively processed to yield the different mature structure. Processing starts with the stepwise removal of the three terminal glucose residues catalysed by two specific glucosidases, and of up to four mannose residues by mannosidases of the endoplasmic reticulum and the cis cisternae of the Golgi apparatus to yield high mannose oligosaccharides. Intermediates destined to become complex type structures are
Table 4 Factors involved in the regulation of oligosaccharide biosynthesis ${ }^{49}$

- conformation of the polypeptide backbone

- physical accessability of oligosaccharide chains

- membrane integration

- expression and substrate specificity of glycosidases and glycosyltransferases

- route and duration of intracellular transport

- location of the glycoprotein in the cell

- subcellular compartmentation of processing enzymes

- concentrations of lipid intermediates (dolichol, retinol)

- concentration of sugar nucleotides

further processed by addition of a $\mathrm{N}$-aceytlglucosamine residue by $\mathrm{N}$-acetylglucosaminyltransferase I followed by removal of two mannose residues by Golgi mannosidase II. Thereafter peripheral sugars are stepwise transferred from sugar nucleotides to the trimmed oligosaccharide in the medial and trans cisternae of the Golgi apparatus before insertion into the plasma membrane. Unlike the assembly of the lipid linked precursor which seems to proceed via a single pathway in most cells, processing of oligosaccharides is tremendously diverse and allows to generate a great variety of oligosaccharide structures.

Whereas the sequence of processing events is fairly well understood, little is known about the control mechanisms that direct the formation of particular structures. Several factors that may control processing have been proposed (Table 4). Whereas the conformation of the polypeptide backbone $e^{57}$ and its insertion into the membrane bilayer $^{58}$ represent determinants which reside in the glycoprotein itself, the concentration of sugar nucleotides and dolicholphosphate ${ }^{59}$ and the level of expression of the various glycosidases and glycosyltransferases may be influenced by endogenous and exogenous stimuli and may reflect conditions at the time of synthesis.

Modifications of oligosaccharides presumedly are not restricted to the biosynthetic pathway, but also seem to occur after insertion of glycoproteins into the plasma membrane. Terminal sugar residues L-fucose and $\mathrm{N}$-acetylneuraminic acid of plasma membrane glycoproteins are rapidly removed from the glycoproteins two to four times faster compared to the half-life of the polypeptide backbone. ${ }^{545560}$ Loss of terminal sugars occurs either in situ in the plasma membrane or in endocytotic compartments during membrane recycling..$^{58}$ Core sugars are removed in the different glycoproteins with individual half-lives in between that of the polypeptide and that of core sugars. ${ }^{54561}$ This indicates that the oligosaccharides of plasma membrane glycoproteins may be trimmed to an individual extent by limited deglycosylation. Knowledge of the pathways and the regulation of oligosaccharide biosynthesis and degradation will not 
only further the discovery of diseases that result from defects in glycoprotein metabolism, but will also provide new therapeutic approaches for treating them.

This work was supported by the Deutsche Forschungsgemeinschaft (SFB 154 and 29).

\section{References}

1 Feizi T, Childs RA. Carbohydrate structures of glycoproteins and glycolipids as differentiation antigens, tumour-associated antigens and components of receptorsystems. Trends Biochem Sci 1985; 10: 24-9.

2 Williams AF. Immunoglobulin-related domains for cell surface recognition. Nature $1985 ; 314$ : 579-80.

3 Neumeier $\mathbf{R}$, Josic $D$, Reutter W. Integral membrane antigens involved in cell substratum adhesion of hepatocytes and hepatoma cells. Exp Cell Res 1984; 151 : 567 72.

4 Lefkowitz RJ, Caron MG, Stiles GL. Mechanisms of membrane-receptor regulation. $N$ Engl J Med 1984; 310: 1570-9.

5 Codogno P, Botti J, Font J, Aubery M. Modification of the $\mathrm{N}$-linked oligosaccharides in cell surface glycoproteins during chick embryo development. Eur $J$ Biochem 1985; 149: 453-60.

6 Ceccarini C, Muramatsu T, Tsang J, Atkinson PH. Growth-dependent alterations in oligomannosyl cores of glycopeptides. Proc Natl Acad Sci 1975; 72: 3139-43.

7 Kato S, Akamatsu N. Alterations in fucosyl oligosaccharides of glycoproteins during rat liver regeneration. Biochem J 1985; 229: 521-8.

8 Hughes RC, Mills G. Analysis by lectin affinity chromatography of N-linked glycans of BHK cells and ricin-resistant mutants. Biochem $J$ 1983; 211 : 575-87.

9 Muramatsu H, Muramatsu T. Decreased synthesis of large fucosyl glycopeptides during differentiation of embryonal carcinoma cells induced by retinoic acid and dibutyryl cyclic AMP. Develop Biol 1982; 90: 441-4.

10 Büchsel R, Reutter W. Plasma membrane changes of liver and Morris hepatoma induced by retinol in rats. Cancer Res 1982; 42: 2450-6.

11 Jogeeswaran G. Cell surface glycolipids and glycoproteins in malignant transformation. Adv Cancer Res 1983; 38: 289-350.

12 Reutter W, Tauber R. Turnover of plasma membrane glycoproteins from liver and hepatoma. GANN 1983; 29: 59-65.

13 Scanlin TF, Wang I, Glick MC. Altered fucosylation of membrane glycoproteins from cystic fibrosis fibroblasts. Pediatr Res 1985; 19 : 368-74.

14 Roelfzema H, Pergers M, Van Erp PEJ, Gommans J M, Mier PD. Studies on the plasma membrane of normal and psoriatic keratinocytes. 4. Characterization of glycoconjugates. Br J Dermatol 1981; 105: 509-16.

15 Ullrich A, Bell JR, Chen EJ, et al. Human insulin receptor and its relationship to the tyrosine kinase family of oncogenes. Nature 1985; 313: 756-61.

16 Kobata A. The carbohydrates of glycoproteins. In: Ginsburg V, Robbins PW, eds. Biology of carbohydrates Vol. 2. New York: Wiley, 1984: 87-161.
17 Finne J. Occurrence of unique polysialosyl carbohydrate units in glycoproteins of developing brain. $J$ Biol Chem 1982; 257: 11966-70.

18 Carrer JP, Brisson J-R. The three dimensional structure of N-linked oligosaccharides. In: Ginsburg V, Robbins PW, eds. Biology of carbohydrates Vol. 2. New York: Wiley, 1984: 289-331.

19 Gerok W, Köttgen E, Reutter W. Glycoproteins on hepatocytic surfaces. In: Popper H, Schaffner F, eds. Progress in liver diseases. Vol. VII. Grune and Stratton: New York, 1982: 87-107.

20 Olden K, Bernard BA, Humphries MJ, et al. Function of glycoprotein glycans. Trends Biochem Sci 1985; 10: 7882.

21 Burridge $\mathrm{K}$, Jordans L. The glycoproteins of dictyostelium discoideum. Changes during development. Exp Cell Res 1979; 124: 31-8.

22 Edwards JG, Dysart J Mck, Hughes RC. Phenotypic reversion of ricin-resistant hamster fibroblasts to a sensitive state after coating with glycolipid receptors. Nature (London) 1976; 264: 63-6.

23 Hughes RC, Mills G, Stojanovic D. Glycosyltransferases of $\mathrm{N}$-glycan assembly: modulation of activity and its effects on the biological roles of cell surface glycoproteins. In: Popper H, Reutter W, Gudat F, Köttgen E, eds. Structural carbohydrates in the liver. pp. 63-81, MTP Press, Lancaster 1983.

24 Sasak W, Quaroni A, Herscovics A. Changes in cell surface fucose-containing glycopeptides and adhesion of cultured intestinal epithelial cells as a function of cell density. Biochem $J$ 1983; 211 : 75-80.

25 Gesner BM, Ginsburg V. Effect of glycosidases on the fate of transfused lymphocytes. Proc Natl Acad Sci USA 1964 ; 52 : 750-5.

26 Hooghe RJ, Pink JRL. The role of carbohydrate in lymphoid cell traffic. Immunol Today 1985; 6: 180-1.

27 Schlepper-Schäfer J, Kolb-Bachofen V, Kolb $\mathbf{H}$. Analysis of lectin-dependent recognition of desialylated erythrocytes by Kupffer cells. Biochem J. 1980; 186: 827-31.

28 Müller E, Franco MW, Schauer R. Involvement of membrane galactose in the in vivo and in vitro sequestration of desialylated erythrocytes. HoppeSeyler's Z Physiol Chem 1981; 362: 1615-20.

29 Kolb-Bachofen V, Schlepper-Schäfer J, Teradeira R, Vogt D, Kolb H. D-Galactose-specific lectin on rat Kupffer cells-its role in the biology and pathology of the liver. In: Popper H, Reutter W, Gudat F, Köttgen E, eds. Structural carbohydrates in the liver. Lancaster: MTP Press, 1983: 277-86.

30 Schirrmacher V, Altevogt $\mathrm{P}$, Fogel $\mathrm{M}$, et al. Importance of cell surface carbohydrates in cancer cell adhesion, invasion and metastasis. Invasion Metastasis 1982; 2: 313-60.

31 Datema R, Romero PA, Legler G, Schwarz RT. Interference with glycoprotein glycosylation. In: Popper H, Reutter W, Gudat F, Köttgen E, eds. Structural carbohydrates in the liver. Lancaster: MPT Press, 1983: 539-45.

32 Roseman S. The synthesis of complex carbohydrates by multiglycosyltransferase systems and their potential function in intercellular adhesion. Chem Phys Lipids 1970: 5: 270-97. 
33 Pricer WE, Ashwell G. The binding of desialylated glycoproteins by plasma membranes of rat liver. $J$ Biol Chem 1971; 246: 4825-33.

34 Ashwell G, Harford J. Carbohydrate-specific receptors of the liver. Ann Rev Biochem 1982; 51: 531-54.

35 Köttgen E. Lectine. Klin Wochenschr 1977; 55: 35973.

36 Barondes S H. Lectins: their multiple endogenous cellular functions. Ann Rev Biochem 1981; 50: 207-31.

$37 \operatorname{Raz} A$, Lotan R. Lectin-like activities associated with human and murine neoplastic cells. Cancer Res 1981 ; 41 : 3642-7.

38 Edelman GM. Cell adhesion molecules. Science 1983; 219: 450-7.

39 Chen WT, Singer SJ. Immunelectron microscopy studies on the sites of cell-substratum and cell-cell contacts in cultured fibroblasts. J Cell Biol 1982; 95: 205-22.

40 Hynes RO, Yamada KM. Fibronectins multifunctional modular glycoproteins. J Cell Biol 1982; 95: 369-77.

41 Feizi T. Demonstration by monoclonal antibodies that carbohydrate structures of glycoproteins and glycolipids are onco-developmental antigens. Nature 1985; 314: 53-7.

42 Hakomori S. Glycosphingolipids as differentiationdependent, tumor associated markers and as regulators of cell proliferation. Trends Biochem Sci 1984; 9: 4538.

43 Kingsley D, Kozarsky KF, Hobbie L, Krieger M. Reversible defects in O-linked glycosylation and LDL receptor expression in a UDP-Gal/UDP-GalNAc 4epimerase deficient mutant. Cell 1986; 44: 749-59.

44 Kingsley D, Kozarsky KF, Segal M, Krieger M. Three types of low density lipoprotein receptor-deficient mutant have pleiotropic defects in the synthesis of $\mathrm{N}$ linked, O-linked and lipid-linked carbohydrate chains. $J$ Cell Biol 1986; 102: 1576-85.

45 Ronnett GV, Knutson VP, Kohanski RA, Simpson TL, Lane MD. Role of glycosylation in the processing of newly translated insulin proreceptor in 3T3-L1 adipocytes. J Biol Chem 1984; 259: 4566-75.

46 Fambrough DM, Devreotes PN. Newly synthesized acetylcholine receptors are located in the Golgi apparatus. J Cell Biol 1978; 76: 237-44.

47 Merlier JP, Sebbane R, Tzartos S, Linstrom J. Inhibition of glycosylation with tunicamycin blocks assembly of newly synthesized acetylcholine receptor subunits in muscle cells. J Biol Chem 1982; 257 : 2694-701.

48 Sabatini DD, Kreibich G, Morimoto T, Adesnik M. Mechanisms for the incorporation of proteins in mem- branes and organelles. J Cell Biol 1982; 92: 1-22.

49 Kornfeld R, Kornfeld S. Assembly of asparagine-linked oligosaccharides. Ann Rev Biochem 1985; 54: 631-64.

50 Breitfeld PP, Rup D, Schwartz AL. Influence of the Nlinked oligosaccharides on the biosynthesis, intracellular routing and function of the human asialoglycoprotein receptor. J Biol Chem 1984; 259: 10414-21.

51 Reed BC, Lane MD. Insulin receptor synthesis and turnover in differentiating 3T3-Ll preadipocytes. Proc Natl Acad Sci USA 1980; 77: 285-9.

52 Krupp M, Lane MD. On the mechanism of ligand induced down regulation of insulin receptor level in the liver cell. J Biol Chem 1981 ; 256: 1689-94.

53 Olden K, Parent JB, White SL. Carbohydrate moieties of glycoproteins. A re-evaluation of their function. Biochem Biophys Acta 1982; 650: 206-32.

54 Kreisal W, Volk BA, Büchsel R, Reutter W. Different half-lives of the carbohydrate and protein moieties of a 110000 -dalton glycoprotein isolated from plasma membranes of rat liver. Proc Natl Acad Sci USA 1980; 77: 1828-31.

55 Tauber R, Park C-S, Reutter W. Intramolecular heterogeneity of degradation in plasma membrane glycoproteins - Evidence for a general characteristic. Proc Natl Acad Sci USA 1983; 80: 4026-9.

56 Tauber R, Heinze K, Reutter W. Effect of chloroquine on the degradation of L-fucose and the polypeptide moiety of plasma membrane glycoproteins. Eur $J$ Cell Biol 1985; 39: 380-5.

57 Hsieh P, Robbins PW. Regulation of asparagine-linked oligosaccharide processing. J Biol Chem 1984; 259: 2375-82.

58 Tauber R, Schenck I, Josic D, Gross V, Heinrich P C, Gerok W, Reutter W. Different oligosaccharide processing of the membrane-integrated and the secretary form of gp80 in rat liver. Embo $J$ (in press)

59 Carson DD, Earles BJ, Lennarz WJ. Enhancement of protein glycosylation in tissue slices by dolicholphosphate. J Biol Chem 1981; 256: 11552-7.

60 Baumann H, Hou E, Jahreis GP. Preferential degradation of the terminal carbohydrate moiety of plasma membrane glycoproteins in rat hepatoma cells and after transfer to the membranes of mouse fibroblasts. $J$ Cell Biol 1983; 96: 139-50.

61 Volk BA, Kreisel W, Köttgen E, Gerok W, Reutter W. Heterogeneous turnover of terminal and core carbohydrates within the carbohydrate chain of dipeptidylaminopeptidase IV isolated from rat liver plasma membrane. FEBS Lett 1983; 163: 150-2. 